symptoms or visible discharge and $\geq 5 \mathrm{PMNs}$ /high powered field (HPF). Absence of CT, MG, adenovirus, and HSV was considered as idiopathic NGU. Men without NGU had no urethral symptoms, no discharge, and $<5 \mathrm{PMNs} / \mathrm{HPF}$. Broad-range $16 \mathrm{~S}$ rRNA gene PCR with deep sequencing was used to characterize the urethral microbiota. Compositional lasso analysis of bacterial taxa was conducted to identify associations between bacteria and NGU; beta coefficients $(\beta)$ giving change in probability of NGU per $\log 2$ change in relative abundance are reported.

Results Of 434 (199 MSM, 235 MSW) urine samples, 330 yielded sequence data. NGU+ men were less likely to yield sequence data $(70 \%$ vs $84 \%$, Fisher's $\mathrm{p}=0.001)$. Of 328 men with $\geq 1000$ sequence reads/sample, 95 MSM (44 NGU+) and 143 MSW (46 NGU+) were negative for CT, MG, adenovirus, and HSV. Higher relative abundances of Haemophilus influenzae $(\beta=0.0139)$ and Mycoplasma penetrans $(\beta=0.0095)$ were positively associated with idiopathic NGU in MSM, while $H$. influenzae was positively associated with idiopathic NGU in MSW $(\beta=0.0184)$. The model also identified bacterial species that were negatively associated with NGU in MSM and MSW. Notably, Lactobacillus iners was negatively associated with idiopathic NGU in MSW ( $\beta=-0.0006)$ but not MSM.

Conclusion Different bacterial species are associated with NGU in MSM and MSW. We identified two bacterial species infrequently detected in the male urethra as positively associated with NGU. Disclosure No significant relationships.

\section{P524 GAYS, GOVERNMENT AND BIG DATA: SHOULD ROUTINE HEALTH RECORDS INCLUDE SEXUAL ORIENTATION?}

\begin{abstract}
${ }^{1}$ Peter Saxton*, ${ }^{2}$ Jeffery Adams, ${ }^{3}$ John Fenaughty, ${ }^{1}$ Daniel Exeter, ${ }^{4}$ Andrew Sporle. ${ }^{1}$ University of Auckland, School of Population Health, Auckland, New Zealand; ${ }^{2}$ Massey University, ShoreWhariki Research Centre, Auckland, New Zealand; ${ }^{3}$ University of Auckland, School of Counselling, Human Services and Social Work, Auckland, New Zealand; ${ }^{4}$ University of Auckland, Department of Statistics, Auckland, New Zealand
\end{abstract}

\subsection{6/sextrans-2019-sti.602}

Background Sexual orientation minorities continue to experience poorer outcomes in sexual health, mental health and addictions. Despite clear information needs, routine data identifying gay, lesbian and bisexual (GLB) individuals are seldom collected by governments, rendering these populations invisible. In New Zealand (NZ), everyone is assigned a unique National Health Index (NHI) number used across all health systems to improve clinical and public health decision-making. In 2017 the NZ Ministry of Health proposed adding sexual orientation and other fields to NHI, however sexual orientation was rejected after consultation. We sought to better understand these viewpoints.

Methods We used the Official Information Act (OIA) to request the complete list of submitters, their support or opposition for adding sexual orientation and written comments. We allocated submitters to six groupings: Government $(G)$; Health provider including District Health Boards (H); NGO or civil society (N); Academic (A); Data management firm (D) or Unspecified (U). Submitters did not necessarily represent the official views of their organisation. We present descriptive summaries and feedback themes.
Results 130 submissions were received. Overall 27 supported sexual orientation in NHI and 35 were opposed, the remainder being neutral or conditional. Support by grouping (high to low) was: Academic (100\%); Government (56\%); Unspecified (44\%); NGO (40\%); Health (36\%) and Data firm (25\%). Supportive reasons included: service planning; evidence-based policy; equity; GLB-specific health delivery (e.g. HPV vaccines, HIV and STI screening, pre-exposure prophylaxis); normalisation; health workforce development. Opposing reasons included: sexual orientation being irrelevant to clinical decision-making; classification challenges (e.g. sexual orientation fluidity); data quality; privacy; discrimination.

Conclusion Barriers to sexual orientation data collection include practical concerns but also well-meaning paternalism and heteronormative assumptions. Better information about NHI uses and protections, data governance, and healthcare service obligations to GLB communities, may improve support. Disclosure No significant relationships.

\section{P525 PREVALENCE OF MYCOPLASMA GENITALIUM BY ANATOMICAL SITE IN MEN WHO HAVE SEX WITH MEN: A SYSTEMATIC REVIEW AND META-ANALYSIS}

${ }^{1}$ Rosie Latimer*, ${ }^{2}$ Hannah Shilling, ${ }^{1}$ Lenka Vodstrcil, ${ }^{2}$ Dorothy Machalek, ${ }^{3}$ Christopher Fairley, ${ }^{1}$ Tim Read, ${ }^{3}$ Eric Chow, ${ }^{3}$ Catriona Bradshaw. 'Monash University, Central Clinical School, Carlton, Australia; ${ }^{2}$ The Royal Women's Hospital, Centre for Women's Infectious Disease Research, Parkville, Australia; ${ }^{3}$ Alfred Health, Melbourne Sexual Health Centre, Carlton, Australia

\subsection{6/sextrans-2019-sti.603}

Background With the current debate over testing and screening for Mycoplasma genitalium (MG) in various populations, more information on the prevalence of $\mathrm{MG}$ is needed particularly in populations at high risk of sexually transmitted infections, such as men who have sex with men (MSM). We assessed the available data on the prevalence of MG in MSM across three anatomical sites: the urethra, pharynx and rectum.

Methods Ovid Medline, PubMed, Embase were searched for all peer-reviewed studies published until 1st June 2018 (in addition to conference proceedings from 2015), that reported prevalence of MG (using nucleic acid amplification testing) in the urethra, rectum and/or pharynx in at least 50 MSM. Data were extracted by anatomical site, symptom and HIV status. Pooled estimates (95\% confidence intervals [CIs]) were calculated using random effects meta-analysis. Subgroup analyses were performed to assess heterogeneity between studies.

Results Forty-six studies met inclusion criteria. The overall prevalence of $\mathrm{MG}$ at any site was $5.8 \% \quad(4.5-7.3 \%$, $\mathrm{I} 2=95.0 \%)$. MG prevalence was 4.6\% (3.0-6.4\%, I2=94.4\%) in the urethra, $6.1 \%(4.5-7.9 \%, \mathrm{I} 2=89.0 \%)$ in the rectum, and $1.0 \%(0.0-5.1 \%, \mathrm{I} 2=96.0 \%)$ in the pharynx. Pooled estimates of MG prevalence were higher among HIV-positive compared with HIV-negative men $\mathbf{( 9 . 0 \%}$ [5.2-13.4\%, $\mathrm{I} 2=90.7 \%]$ versus $5.7 \% \quad[3.5-8.2 \%, \mathrm{I} 2=93.1 \%], \mathrm{p}=0.019)$, and among symptomatic men compared to asymptomatic men $(9.2 \% \quad[6.2-12.7 \%, \quad \mathrm{I} 2=87.3 \%]$ versus $4.0 \% \quad[2.3-6.2 \%$, $\mathrm{I} 2=90.7], \mathrm{p}=0.003)$.

Conclusion MG is commonly detected in MSM, particularly in the urethra and rectum. Prevalence was highest in HIV 